\title{
New implementation of second harmonic generation microscopy for three-dimensional resolution
}

Changhuei Yang, Jerome Mertz

Changhuei Yang, Jerome Mertz, "New implementation of second harmonic generation microscopy for three-dimensional resolution," Proc. SPIE 4963, Multiphoton Microscopy in the Biomedical Sciences III, (10 July 2003); doi: $10.1117 / 12.478032$

SPIE. Event: Biomedical Optics, 2003, San Jose, CA, United States 


\title{
New implementation of second harmonic generation microscopy for three-dimensional resolution
}

\author{
Changhuei Yang*, Jerome Mertz \\ Laboratoire de Neurophysiologie et Nouvelles Microscopies, Ecole Supérieure de \\ Physique et Chimie Industrielles, Institut National de la Santé et de la Recherche \\ Médicale (INSERM EPI-0002), Centre National de Recherche de la Recherche \\ Scientifique (CNRS FRE-2500), 10 rue Vauquelin, 75005 Paris, France
}

\begin{abstract}
We present a fast scanning transmission-mode confocal scanning laser microscope system based on the use of a second harmonic generation (SHG) crystal for signal detection. The quadratic intensity dependence of SHG is exploited to preferentially reveal unscattered signal light and reject out-of-focus scattered background. The SHG crystal plays the role of a virtual pinhole that remains self-aligned without a need for de-scanning. We demonstrate that this new microscope method produces images with higher contrast and less speckle than transmission scanning microscopy with linear detection.
\end{abstract}

Keywords: second harmonic, confocal, microscopy

\section{INTRODUCTION}

Second harmonic generation microscopy [1-3] uses nonlinear optics to extract information from a biological sample. The characteristics of second harmonic generation, namely its sensitivity to phase matching and its nonlinear dependency on the input light intensity, leads to interesting and potentially useful applications in biomedical optics imaging that cannot be accessed by fluorescence. We present one such application in this manuscript. In this application, we exploit the nonlinear conversion property of second harmonic generation to construct a fast scanning confocal microscope that operates in the transmission mode.

Confocal scanning laser microscopy (CSLM) is based on the use of a pinhole in the detection path to provide 3-dimensional image resolution and enhanced background rejection $[4,5]$. In the usual CSLM implementation, detected light is de-scanned so that the pinhole effectively tracks the position of the laser focus at the sample. Such de-scanning is readily accomplished in a reflection configuration by retracing the signal path through the laser scanning optics. In a transmission configuration, however, de-scanning is technically much more difficult and typically requires the use of a second synchronized scanning system [1] or of an elaborate beam path to re-direct the transmitted light into the backward direction [6,7]. The nonlinear intensity dependence of second-harmonic generation allows us to create a virtual pinhole that automatically tracks the laser transmission. The principle is explained below.

\section{THEORY AND EXPERIMENT}

In standard transmission CSLM, laser light transmitted through the sample is focused onto a pinhole of area $A_{p}$ before detection. If the transmitted light has power $P$ and is distributed over a characteristic area $A$ at the pinhole plane, the detected power scales as $P A_{p} / A$ (assuming $A_{p}<A$ ). In our method, the pinhole is replaced by a thin nonlinear crystal and only SHG is detected. Because SHG scales quadratically with incident intensity, the resulting signal scales approximately as $P^{2} / A$. In both cases, the detected signal scales inversely with $A$, implying that out-of-focus light at the aperture (ie. crystal) plane is rejected. A 
distinct advantage of using a SHG crystal instead of a pinhole is that it has a large area, allowing it to play the role of an aperture even when the transmitted signal light is not de-scanned. That is, the crystal may be thought of as a self-aligned virtual pinhole.

We demonstrate the above "virtual pinhole" principle with the experimental setup shown in Fig. 1. We use a mode-locked Ti:Sapphire laser (Spectra-Physics) to generate laser pulses at 860nm wavelength, $\sim 100 \mathrm{fs}$ duration, and $82 \mathrm{MHz}$ repetition rate, that are focused into a target sample with a $60 \times 0.9$ N.A. waterimmersion objective (Olympus; focal waist $w_{0}=0.5 \mu \mathrm{m}$ ). The transmitted light is collected with an identical objective and refocused onto a Type I, $200 \mu \mathrm{m}$ thick lithium triborate crystal (Castech). The total magnification factor, $M$, from the sample to the crystal is approximately 30 , leading to a confocal parameter at the crystal of about $800 \mu \mathrm{m}$ (ie. the crystal is thin relative to this confocal parameter). The laser power incident on the sample, $P_{0}$, is typically $10 \mathrm{~mW}$. The laser beam is raster scanned in the $x-y$ direction with galvanometer mounted mirrors, and the sample is scanned in the $z$ direction with a motorized translation stage.



Figure 1: Experimental layout. MO = microscope objective. Filter transmits only SHG light.

The preferential detection of image bearing light with our virtual pinhole technique can be understood using the following simple argument based on the SHG process and the characteristics of different types of light transmitted through a scattering medium. The relation between the light incident on the SHG crystal with intensity $I(r, \theta) \quad(r, \theta$ are coordinates on the en-face plane of the crystal) and the resultant SHG power is given by:

$S H G \propto \int(I(r, \theta))^{2} r d r d \theta$

From Eq. (1), it is clear that for a given incident light power, the tighter the power is focused onto the crystal, the stronger the resultant SHG signal becomes. For our purpose, the light transmitted through a scattering medium can be classified into four components. The first is the unscattered transmission. This component is focused to a tight spot of Gaussian profile on the SHG crystal. The presence of an absorber or scatterer in the focal volume within the sample provokes an attenuation of this unscattered component, which in turn translates to a shadowgram on the SHG signal. The second type of light is the scattered component originating from the focal volume. Because the crystal is placed in the image plane of the microscope, this component will also focus to a tight spot on the SHG crystal. This component is the direct analogue of the backscattered light used in the conventional reflection mode confocal microscopy. This component and the unscattered component are the two image forming components in the system. The other two components of light are singly scattered light from outside the focal volume and multiply scattered light. These components form diffuse spots on the SHG crystal and thus create correspondingly less SHG signal for a given incident power.

As a demonstration, we consider the signal obtained from a single isolated scatterer, a latex bead, which we scan in the $z$ direction, yielding the axial point-spread-function of our apparatus ( $z=0$ denotes the focal plane). We first note that, in our imaging configuration, the phase of the scattered light at the crystal plane is approximately in quadrature with that of the unscattered light independently of the bead position $z$, as a 
consequence of the cumulative Guoy shifts [8] incurred by both the scattered and unscattered beams before they reach the crystal plane (when $z \gg w_{0}^{2} / \lambda$ the scattered beam incurs no net Guoy shift). To a good approximation, the total intensity incident at the crystal plane is then simply given by the sum of the respective unscattered and scattered intensities:

$$
I(r, z)=P_{0}\left(\left(1-\varepsilon_{z}\right) W_{0}(r)+\varepsilon_{z} \eta W_{S}(r, z)\right)
$$

where, as above, $r$ is the radius from the optical axis at the crystal plane (we assume cylindrical symmetry), $\varepsilon_{z} P_{0}$ is the total power scattered by the bead, $\eta$ is the fraction of this power accepted by the microscope exit pupil (defined here by the collection objective), and $W_{0, S}(r)$ are flux densities normalized so that $2 \pi \int W_{0, S}(r) r d r=1$. These functions allow us to define the characteristic areas

$$
A_{I J}=\left(2 \pi \int W_{I}(r) W_{J}(r) r d r\right)^{-1} \text {. }
$$

Since the SHG produced by the crystal is proportional to $\int I^{2}(r) r d r$, we conclude that

$$
\frac{S H G}{S H G_{0}}=\left(1-\varepsilon_{z}\right)^{2}+2 \varepsilon_{z}\left(1-\varepsilon_{z}\right) \eta \frac{A_{00}}{A_{0 S}}+\varepsilon_{z}^{2} \eta^{2} \frac{A_{00}}{A_{S S}} .
$$
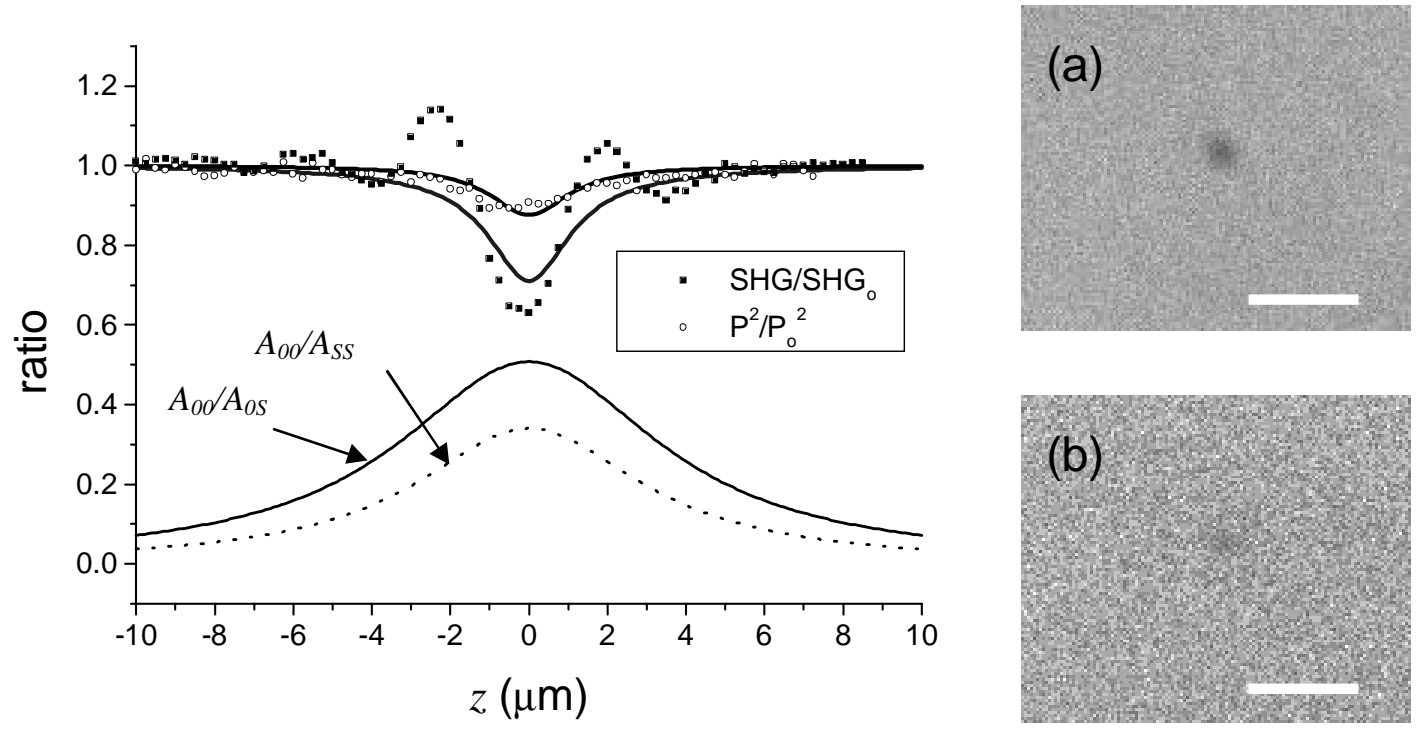

Figure 2: Plots of measured $\langle S H G\rangle / S H G_{0}$ and $\langle P\rangle^{2} / P_{0}^{2}$ for $z$-scan of a $530 \mathrm{~nm}$ latex bead (averaged over 5 scans). Traces are derived from a Gaussian approximation model. Panels illustrate non-averaged SHG (a) and direct $P^{2}$ (b) $x-y$ scan of a bead. Scale bars $=5 \mu \mathrm{m}$.

Several observations are in order. First, $A_{00} / A_{0 S}$ and $A_{00} / A_{S S}$ are smaller than 1 , since $W_{0}(r)$ corresponds to a diffraction limited intensity profile. Second, it is apparent that as far as scattered light is concerned, $A_{00} / A_{0 S}$ and $A_{00} / A_{S S}$ play an aperturing role similar to that of the microscope exit pupil. The smaller the values of $A_{00} / A_{0 S}$ and $A_{00} / A_{s s}$, both of which depend on $z$, the less the scattered light contributes to the SHG signal (ie. the more it is rejected). Finally, for purposes of comparison, we note that if the SHG crystal were removed and the power directly detected and squared, the expression for $P^{2} / P_{0}^{2}$ would be given by Eq. 4 with the replacements $A_{00} / A_{0 S} \rightarrow 1$ and $A_{00} / A_{S S} \rightarrow 1$. In other words, direct detection of power provides no scattered light rejection beyond that of the exit pupil. Figure 2 illustrates $S H G / S H G_{0}$ and $P^{2} / P_{0}^{2}$ for a $z$-scan of a $530 \mathrm{~nm}$ diameter bead. In both cases, the presence of the bead is recognized as a 
reduction in unscattered laser power (first term in Eq. 4). This reduction is undermined by the concurrent detection of forward-directed scattered power that is transmitted through the exit pupil, which we refer to as background (second and third terms in Eq. 4 -- we note that detected singly scattered light originating from the bead, which is normally considered to be signal in a back-reflection geometry, is considered to be background here). Because background rejection is more efficient with the use of the SHG than with direct detection, our method leads to a more highly contrasted bead signal. Moreover, as observed in the bead images (panels in Fig. 2), SHG detection leads to an apparent suppression of speckle noise presumably caused by scattered background

The parameters in Eq. 4 can be roughly estimated in a paraxial approximation by assuming that $W_{0}(r)$ and $W_{S}(r, z)$ are Gaussian in profile, leading to $A_{00} \approx M^{2} \pi w_{0}^{2}, A_{S S} \approx M^{2} \pi w_{S}^{2}\left(1+\left(\lambda z / \pi w_{S}^{2}\right)^{2}\right)$, and $A_{0 S}=\left(A_{00}+A_{S S}\right) / 2$, where $w_{S}$ is the effective bead radius as it appears through the microscope exit pupil. The scattering parameter $\varepsilon_{z}$ is dependent on $z$ since it depends on the laser intensity incident on the bead. Denoting $\sigma$ as the bead scattering cross-section, then $\varepsilon_{z} \approx \sigma / U_{z}$, where $U_{z}=\frac{1}{2} \pi w_{0}^{2}\left(1+\left(\lambda z / \pi w_{0}^{2}\right)^{2}\right)$ is the effective area of the laser beam at the axial position $z$. The pupil transmission $\eta$, on the other hand, is approximately independent of $z$ for small $z$ 's. The following estimates are derived from Mie theory [9]: $\sigma \approx \pi \times(0.15 \mu m)^{2}$, $w_{S} \approx 0.83 \mu \mathrm{m}$, and $\eta \approx 0.75$. As is evident from a comparison with experimental data, our Gaussian approximation is overly simplistic and cannot account for the observed ringing in the SHG trace, presumably caused by the sharp cut-off in the pupil transmission. Nevertheless, it illustrates a salient principle of our microscopy technique, namely that $A_{00} / A_{0 S}$ and $A_{00} / A_{S S}$ are smaller than 1 , leading here to an improvement in signal contrast with SHG detection.

To demonstrate that virtual pinhole microscopy with SHG detection also leads to improved out-of-focus background rejection, we acquire a $z$-stack of $x-y$ scans of a slab of $1 \mu \mathrm{m}$ latex beads suspended in $0.3 \%$ agarose (number concentration $N=0.0071 \mu \mathrm{m}^{-3}$; slab thickness $L=170 \mu \mathrm{m}$ ). Since $\varepsilon_{z}$ fluctuates randomly for different $x-y-z$ positions in the slab, we write $\varepsilon_{z}=\left\langle\varepsilon_{z}\right\rangle+\delta \varepsilon_{z}$, where the brackets refer to the average over an ensemble of $x-y$ scans. If the scattering beads are randomly distributed in the slab and $\delta z$ is chosen large enough so that $\varepsilon_{z}$ and $\varepsilon_{z+\delta}$ are uncorrelated, then $\left\langle\varepsilon_{z}\right\rangle \approx N \sigma \delta z$ and $\left\langle\delta \varepsilon_{z}^{2}\right\rangle \approx N \sigma^{2} \delta z / U_{z}$. Though these last expressions require $\sigma \ll U_{z}$, meaning their validity breaks down somewhat in the immediate vicinity of the focal plane, we infer that $\left\langle\varepsilon_{z}\right\rangle,\left\langle\delta \varepsilon_{z}^{2}\right\rangle<<1$ throughout most of the sample. Eq. (4) then leads to the approximation:

$$
\frac{\langle S H G\rangle}{S H G_{0}} \approx \prod_{z=z_{\text {sut }}-L / 2}^{z_{w \mu H}+L / 2}\left(1-2\left\langle\varepsilon_{z}\right\rangle\left(1-\eta_{z} \frac{A_{00}}{A_{0 S}}\right)+\left\langle\delta \varepsilon_{z}^{2}\right)\left(1-2 \eta_{z} \frac{A_{00}}{A_{0 S}}+\eta_{z}^{2} \frac{A_{00}}{A_{S S}}\right)\right),
$$

where $z_{\text {slab }}$ is the axial location of the slab center and $\eta_{z}$ is no longer assumed to be constant since $|z|$ can be large. Expression 5 is readily evaluated with the substitution $\prod_{z}(1-f(z) \delta z) \approx \exp \left(-\int_{z} f(z) \delta z\right)$, yielding

$$
\frac{\langle S H G\rangle}{S H G_{0}} \approx \exp \left(-2 N \sigma \int_{z_{i, \omega}-L / 2}^{z_{\text {iut }}+L / 2}\left(1-\eta_{z} \frac{A_{00}}{A_{0 S}}-\frac{\sigma}{2 U_{z}}\right) \delta z\right),
$$

where only the dominant terms have been kept. Relation 6 can be analytically expressed when using the Gaussian approximation. In particular, we can assume that $\eta_{z}$ is approximately constant within the range of the integration where $A_{00} / A_{0 S}$ is significant: 


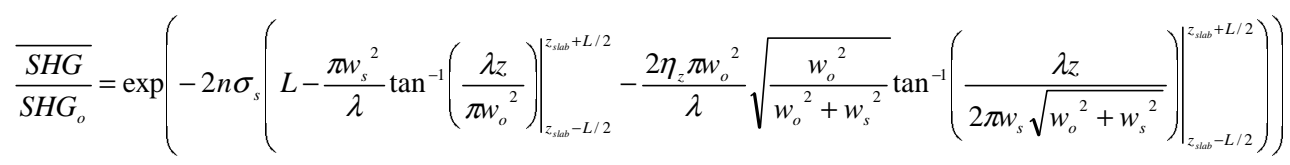

Again for comparison, we note that in the case of direct detection

$$
\frac{\langle P\rangle^{2}}{P_{0}^{2}} \approx \exp \left(-2 N \sigma \int_{z_{s a b}-L / 2}^{z_{s a b}+L / 2}\left(1-\eta_{z}\right) \delta z\right) .
$$

In this case, the $A_{00} / A_{0 S}$ term is absent, and hence $\eta_{z}$ cannot be assumed to be constant over the entire integration range.

From the equations, we observe that SHG detection is sensitive to $\delta \varepsilon_{z}^{2}$ (manifested by the $U_{z}$ dependence), whereas direct detection is not.

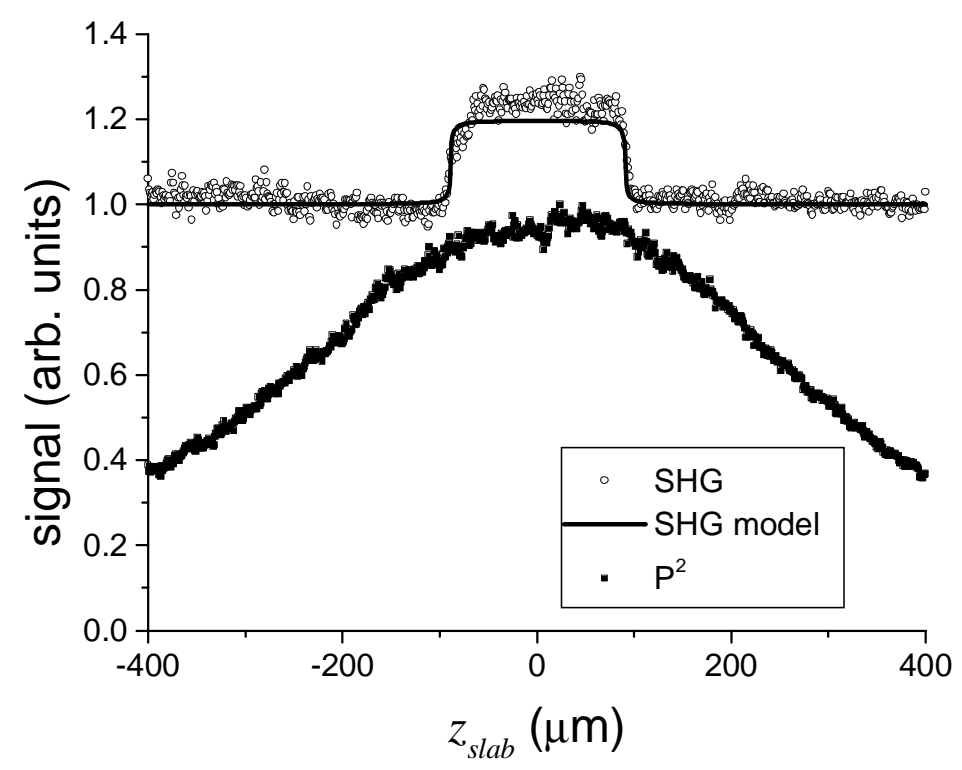

Figure 3: Plots of measured $\langle S H G\rangle$ and $\langle P\rangle^{2}$ for a $z$-scan of a $170 \mu \mathrm{m}$ thick agarose slab of $1 \mu \mathrm{m}$ latex beads, and theoretical trace derived from Gaussian approximation, normalized to arbitrary units.

Figure 3 illustrates both SHG and direct detection signals, averaged over $x-y$, for different values of $z_{\text {slab }}$.

The qualitative difference in the traces is striking. The large but gradual increase in $\langle P\rangle^{2}$ as the slab approaches the focal plane indicates that a significant fraction of the transmitted power consists of out-offocus scattered light. This is expected from the fact that the scattering is mostly forward directed $\left(\eta_{z} \approx 0.9\right.$ near the focal plane). As is manifest from Fig. 3, the slab displacement must be quite large $\left(\left|z_{\text {slab }}\right|>400 \mu \mathrm{m}\right)$ before scattered light is significantly rejected by the exit pupil. In contrast, out-of-focus scattered light is much more efficiently rejected when using SHG detection because $A_{00} / A_{0 S}$ tends towards zero for relatively small displacements from the focal plane (see Fig. 2). When complete rejection is achieved, only unscattered light produces signal, and $\langle S H G\rangle$ and $\langle P\rangle^{2}$ are both proportional to $e^{-2 N \sigma L}$, which is $z^{-}$ 
independent. The apparent plateau in the SHG trace stems from the fact that $A_{00} / A_{0 S}$ and $\delta \varepsilon_{z}^{2}$ are nonnegligible only when the slab spans the focal plane. This plateau clearly identifies the slab boundaries, demonstrating the advantage of improved out-of-focus background rejection with SHG detection.

Equation 6 predicts that the ratio of the signal plateau height to the baseline level should increase linearly with increasing sample turbidity. This in turn signifies that the out-of-focus background rejection improves with sample turbidity. This prediction was experimentally verified (see Fig. 4), and stems from the sensitivity of our SHG detection to $\delta \varepsilon_{z}^{2}$.

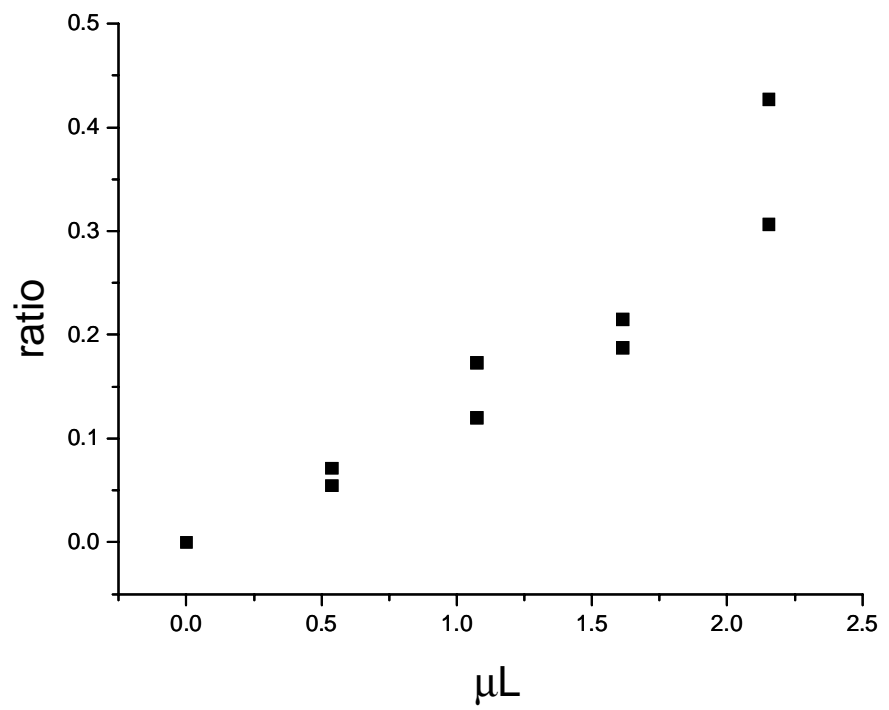

Figure 4: Plots of ratio of the measured $\langle S H G\rangle$ plateau peak to its baseline for a $z$-scan of a $170 \mu \mathrm{m}$ thick agarose slab of $1 \mu \mathrm{m}$ latex beads, for different bead concentration. $\mu$ is the scattering coefficient.
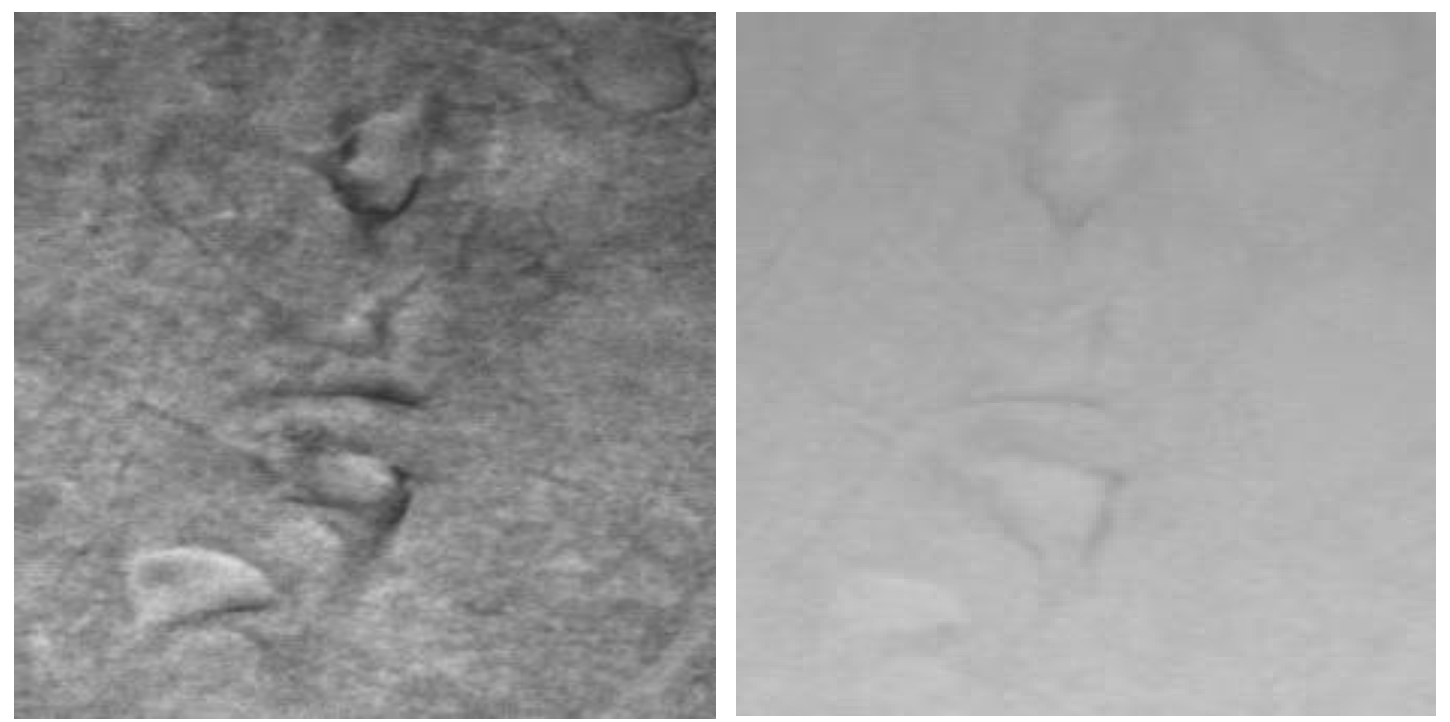

Figure 5: $x-y$ images of a rat brain section using SHG detection (left), and direct $P^{2}$ detection (right). 


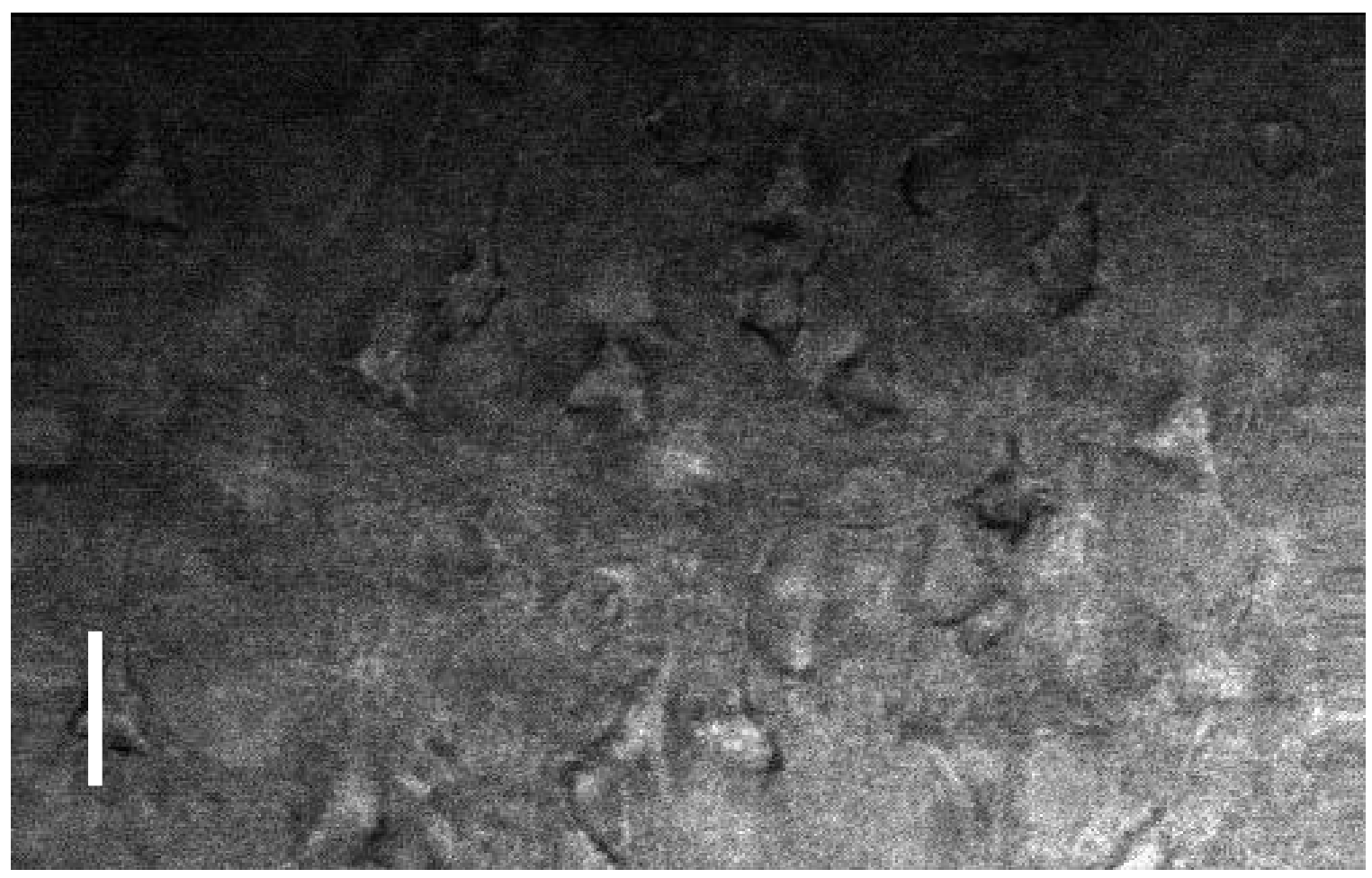

Figure 6: $x-y$ images of a rat brain section using SHG detection. The scanned region is cortical layer 5 and an abundance of pyramidal cells can be observed. Scale bar is 50 microns long.

\section{APPLICATION}

Finally, for purposes of illustration, we use our virtual pinhole technique to image a rat brain section at a depth of 50 microns. The $\langle S H G\rangle$ image and the corresponding $\langle P\rangle^{2}$ image are shown in Fig 5. The $\langle S H G\rangle$ image shows a greater contrast. A larger $\langle S H G\rangle$ image of the cortical layer 5 region of the brain is shown in Fig. 6.

\section{CONCLUSIONS}

In conclusion, we have demonstrated a new implementation of transmitted light CLSM where an SHG crystal serves as a self-aligned virtual pinhole. Because the SHG signal scales inversely with the area of the incident light distribution, it preferentially reveals unscattered (focused) rather than scattered (diffuse) transmitted power. We emphasize that our technique works well provided an adequate supply of unscattered light survives transmission through the sample. The fact that unscattered power decays exponentially with sample thickness imposes limits on the technique's applicability. In particular, for thick samples, SHG signal from unscattered light can easily be dominated by SHG from scattered background, despite the suppression of the latter by the virtual pinhole effect. We have empirically observed, with samples comprising $1 \mu \mathrm{m}$ beads, that our technique is effective up to sample thicknesses of roughly $3 / N \sigma$ (ie. 3 scattering lengths).

A notable advantage of our technique lies in its ease of implementation, particularly in combination with standard two-photon excited microscopy [10], which can be operated simultaneously. Finally, we note that our technique is not limited to signal conversion with an SHG crystal. Alternative techniques involving, for example, 2-photon excited fluorescence or wide-bandgap semiconductors could achieve similar virtual pinhole effects.

We thank L. Moreaux for initial work on this project. C. Y. is grateful to INSERM(France) and ASTAR(Singapore) for financial support and fellowship award. 


\section{REFERENCES}

1. Georgiou, E., et al., Second and third optical harmonic generation in type I collagen, by nanosecond laser irradiation, over a broad spectral region. Optics Communications, 2000. 176(13): p. 253-260.

2. Guo, Y.C., et al., Second-harmonic tomography of tissues. Optics Letters, 1997. 22(17): p. 13231325.

3. Freund, I., M. Deutsch, and A. Sprecher, Connective-Tissue Polarity - Optical 2nd-Harmonic Microscopy, Crossed-Beam Summation, and Small-Angle Scattering in Rat-Tail Tendon. Biophysical Journal, 1986. 50(4): p. 693-712.

4. Wilson, T. and C. Sheppard, Theory and Practice of Scanning Optical Microscopy. 1984, London: Academic press.

5. $\quad$ Minsky, M., Microscopy Apparatus. 1957: USA.

6. Dixon, A.E., S. Damaskinos, and M.R. Atkinson, A Scanning Confocal Microscope for Transmission and Reflection Imaging. Nature, 1991. 351(6327): p. 551-553.

7. Brakenhoff, G.J., Imaging Modes in Confocal Scanning Light-Microscopy (Cslm). Journal of Microscopy-Oxford, 1979. 117(NOV): p. 233-242.

8. Born, M. and E. Wolf, Principles of Optics. 1999, Cambridge: Cambridge University Press.

9. Bohren, C. and D. Huffman, Absorption and scattering of light by small particles. 1983, New York: Wiley-Interscience.

10. Denk, W., J.H. Strickler, and W.W. Webb, 2-Photon Laser Scanning Fluorescence Microscopy. Science, 1990. 248(4951): p. 73-76.

*kelpkeeper@alum.mit.edu; phone 1919 660-2475; fax 1919 660-2476 